\title{
Transient-based automatic incident detection method for intelligent transport systems
}

\author{
Attila M. Nagy, Bernát Wiandt and Vilmos Simon
}

\begin{abstract}
One of the major problems of traffic in big cities today is the occurrence of congestion phenomena on the road network, which has several serious effects not only on the lives of drivers, but also on city inhabitants. In order to deal with these phenomena, it is essential to have an in-depth understanding of the processes that lead to the occurrence of congestion and its spilling over into contiguous areas of the city.

One of the main causes of congestion phenomena is unexpected traffic incidents on major roads and urban freeways, the rapid and reliable detection of which can help reduce negative impacts. Researching Automatic Incident Detection (AID) has a long history that has again become one of the main subjects of research with the rise of new machine learning methods.

Our article presents a new Transient-based Automatic Incident Detection (TBAID) method we have developed, which uses an approach not yet seen in professional literature to detect the occurrence of incidents. The results of our detailed analysis showed that our method performed better than the methods currently available in terms of both speed and reliability on traffic data collected from freeways.

We also created a new dataset for the examination of our method, because the datasets used in previous research were either too small or not publicly available. Our dataset contains 452 incidents and data measured with dual loop traffic detectors from the immediate vicinity of incidents, which, to the best of our knowledge, is the largest publicly available incident dataset to date.
\end{abstract}

Index Terms - automatic incident detection, time series analysis, congestion, smart cities

\section{INTRODUCTION}

One of the major problems related to transportation in major cities around the world is the phenomenon of traffic jams and congestion occurring on major roads and urban freeways. Congestion has a serious impact not only on the lives of vehicle drivers, but also on the lives of every inhabitant of the city. Congestion increases energy and fuel consumption, as well as harmful emissions [1], [2]. Other research has focused on the physiological effects of congestion. Air pollution associated with congestion has been shown to increase the chances of developing allergies [3] and to aggravate the symptoms of people who are sensitive to them. In addition, studies have shown that congestion also increases the risk of heart attacks [4].

Attila M. Nagy is with the Department of Networked Systems and Services, Faculty of Electrical Engineering and Informatics, Budapest University of Techonolgy and Economics, Hungary, e-mail: anagy@hit.bme.hu.

Bernát Wiandt is with the Department of Networked Systems and Services, Faculty of Electrical Engineering and Informatics, Budapest University of Techonolgy and Economics, Hungary, e-mail: bwiandt@hit.bme.hu.

Vilmos Simon is with the Department of Networked Systems and Services, Faculty of Electrical Engineering and Informatics, Budapest University of Techonolgy and Economics, Hungary, e-mail: svilmos@hit.bme.hu.
The negative effects listed above illustrate the significance of avoiding and possibly eliminating congestion, as they harm the health of citizens in addition to causing significant economic damage. A reduction of congestion would bring serious economic and social benefits [5].

Intelligent city management systems can provide solutions to these problems or at least significantly reduce negative impacts with the help of Intelligent Transportation Systems (ITS) [6]. The task of these systems is to continually monitor the traffic and to provide information to the urban transport infrastructure designers and operators based on the collected data, as well as to manage the automated allocation of resources, for example, opening or closing new lanes, adapting traffic lights to current traffic conditions [7] or assisting route planning applications with accurate forecasts.

There are countless reasons for the occurrence of congestion, only some of which can be predicted. Unpredictable congestion phenomena typically do not repeat and are usually caused by unexpected traffic incidents. Research has shown that traffic incidents account for at least $60 \%$ of non-recurring congestion [8], [9].

In order to reduce the negative effects of unexpected traffic incidents, it is essential that intelligent city management systems are able to respond as quickly as possible to unexpected situations. In addition to providing useful information to city traffic management, quick and reliable AID can also provide new data for route planning and traffic forecasting algorithms, along with being an important data source for dynamic traffic light control systems.

AID is a long-established area of research that has come back into focus now that new types of data sources and data analysis methods, as well as increasingly used artificial intelligence-based solutions, have become wide-spread [10]. From the start, it has been a major challenge for researchers to address the contradiction between the accuracy and the speed of detection. Looking at the performance of the methods found in professional literature, it can be concluded that although the methods are capable of high detection rates, even close to $100 \%$, they are also very slow to detect incidents, or they send a number of false alarms. The opposite of this phenomenon can also be observed, with rapid detection being achieved but accompanied by a low accuracy of less than $70 \%$. It is important to point out that frequent false detection makes the task of traffic management extremely difficult. False detection can result in incorrect reallocation of resources and modification of traffic light schedules, which can upset the otherwise normal pace of traffic.

Another major challenge with AID is obtaining a suitable 
dataset. Since incidents are rarely occurring events, collection is difficult in large quantities. It is also important that we have information not only about incidents, but also about traffic data in their immediate vicinity. Unfortunately, the datasets used for research in professional literature contain a small number of incidents (10-30), which is not sufficient for the artificial intelligence models used today, or the dataset has not been made publicly available.

In this article, we would like to offer a solution to the two challenges mentioned above. First, we created an incident dataset containing data from 452 incidents as well as traffic detector data from the immediate vicinity of incidents for the investigated time period. The dataset has been made publicly available to make our results reproducible and to assist related scientific research.

Using the completed dataset, we developed a new AID model. To do this we applied a new approach, in which we used state-of-the-art machine learning tools and developed new, complex features that focus on detecting transient phenomena caused by incidents in traffic data. Our detailed analysis showed that the model we developed can surpass the methods from professional literature in accuracy as well as speed, with low false alarm rates.

The remainder of this article contains the following sections. In Section II, we present related works found in professional literature. In addition to presenting previously developed AID methods, we also place considerable focus on describing the key features of incidents. The method we have developed is described in Section III. The evaluation of TBAID is performed in Section IV, where we compare it with the results of several machine learning models and previous AID methods. We end our article with a short conclusion in Section $\mathrm{V}$.

\section{RELATED RESEARCH}

For decades, researchers and city managers have been working on ways to automate the detection of traffic incidents. The reliable and fast Automatic Incident Detection (AID) allows city managers to take preventive action to avoid congestion, as well as route planners and forecasting systems to use this additional information to improve planned routes and forecasts.

The proper implementation of incident detection requires understanding and examination of traffic phenomena caused by incidents. Therefore, in Section II-A we focus on presenting the features defined by professional literature that are currently used for implementing AID. The main AID methods from professional literature are then described in Section II-B.

\section{A. Incidents}

An incident is defined as any non-recurring event on a road network that reduces the capacity of a given road segment. An incident can be an accident, a pulled over or broken down vehicle, traffic hazards, debris on the road, fallen cargo, road network maintenance or refurbishment and other special, nonemergency events [11], [9]. Events in the previous list are referred to as incident types.
To categorize incidents, the Traffic Incident Management Handbook (TIMH) [12] defines an incident profiling and classification procedure based on the type, location (has it blocked a lane?) and duration of the incident. The incident classification shows that we have data for $70 \%$ of all incidents, of which $80 \%$ are related to vehicles pulled over, $10 \%$ are accidents and the remaining $10 \%$ are classified in other categories. It can be seen that in all cases the incidents that block lanes are causing relatively large delays, but the incidents at the side of the road can also cause measurable capacity reductions. Accidents blocking multiple lanes cause considerably large delays. This means that incidents that have no effect on traffic development cannot be detected from traffic data, so there will definitely be a subset of incidents that are impossible to detect with a traffic-data based AID.

The Manual on Uniform Traffic Control Devices (MUTCD) [13] compiles incident types into three main categories based on a similar set of criteria. Major incidents last for at least 2 hours and are typically fatal accidents or other incidents involving dangerous substances that are difficult to clean getting on the road. When this happens, it is often necessary to close all lanes (interestingly, these 2 hours are not in line with the maximum 90-minute value in TIMH [12]). The length of intermediate incidences falls between 30 minutes and 2 hours. This may require the complete closing of the given road segment, but partial roadblocks are more common. Minor incidences are those of less than 30 minutes that rarely require lane closure. Typically this includes vehicles that are pulled over or small collisions.

Recently, the length of incidents has been the subject of several studies [14], [15], [16], as this may be valuable information for road network management organizations, route planning algorithms or traffic forecasting services. These researches have found that different types of incidents have different lengths that correspond to them. A study conducted in Australia [15] showed that the accidents included in the study lasted on average 43 minutes and the incidents related to pulled-over vehicles lasted on average 41 minutes. Hazards have the longest lasting effects, with an average length of 74 minutes. Another interesting observation was that incidents last longer on weekdays than on weekends.

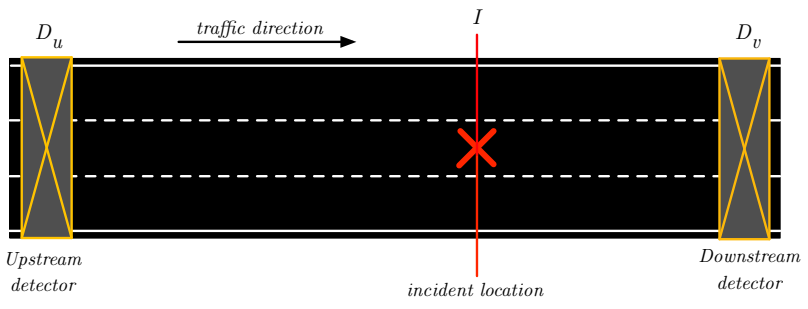

Fig. 1: Configuration method for examining traffic incidents.

A common examination method for traffic incidents is illustrated in Figure 1, where incidents are detected with data from traffic detectors [17], [18], [19], [20], [21]. Of course, there are other methods [22], [23], which determine the occurrence of an incident from travel time data or speed/acceleration data 
Transient-based automatic incident detection

method for intelligent transport systems extracted from vehicle trajectories, but we won't deal with these approaches here.

Incident $I$ is detected using two traffic detectors: $D_{u}$ (upstream) and $D_{d}$ (downstream). In relation to traffic direction, the upstream detector is located in the pre-incident area, while the downstream detector is monitoring traffic in the postincident segment. Using upstream and downstream detectors, several articles break down the investigated road network into segments, where detection is carried out.

The detectors typically measure flow, speed and occupancy values, which are always determined for consecutive time intervals of a fixed length. Typical time intervals include 30 seconds, 5 minutes and 1 hour. Flow represents the number of vehicles per time unit (veh/h) and speed represents the average speed of vehicles passing by a detector for a given time interval. Occupancy indicates the percentage of time vehicles were over a detector for a given time interval.

In case of incident $I$, different phenomena may be observed on the upstream and downstream detectors [24].

In order to better illustrate the differences in the forming traffic patterns between upstream and downstream detectors, a comparison of different measured metrics can be found in Figure 2 for three different traffic demands. If, as a result of the incident, the capacity of the affected road segment is reduced at the location of the incident, vehicles will start to pile up when traffic demand rises above this amount on the pre-incident segment towards the upstream detector. As soon as the effect reaches the $D_{u}$ upstream detector, significantly reduced flow and speed values can be measured (Figure 2c and Figure 2e), while occupancy, in contrast, increases (Figure 2a). However, it is important to note that if the traffic demand is low enough, it is impossible to detect the incident from traffic detector data, since even with reduced capacity, the road segment can serve the current traffic demand.

In the meantime, the measured speed values on the $D_{d}$ downstream detector start to increase up to the free-flow speed (Figure 2d).

Free-flow speed is the speed at which vehicle drivers can go when other vehicles do not impede their movement [25]. The vehicles are congested before the incident, therefore the flow and occupancy values measured on the downstream detector will show a declining trend compared to the preincident values (Figure $2 f$ and Figure $2 b$ ). Another important observation is that the effect on the upstream detector appears slower than on the downstream detector, as the congestion phenomenon propagating in the upstream direction is moving slower than the vehicles leaving the incident.

As a first step we looked at the occupancy time series. Figure 2a shows a dramatic increase in occupancy in case of all three traffic demands after the occurrence of the incident with variable delay. An interesting observation of the upstream is that the time it takes for the effect of the incident to appear depends on the traffic demand. The higher the traffic demand, the faster the effect appears. This is logical, since vehicles are congesting faster behind each other.

It is important to note that depending on the position of the incident between two measuring stations, the effect of the incident on the detectors appears with different delays.
In contrast to upstream, the phenomenon that appears downstream (Figure $2 b$ ) appears quite quickly, but even though the effect can be detected it is less distinct than in the case of upstream. The time of the effect appearing downstream does not depend on traffic demand.

A drastic change, much like upstream occupancy, can be seen in Figure 2c, which shows upstream speeds. After the incident, the measured speed started to decrease sharply with the delay, depending on the traffic demand. As seen in Figure $2 \mathrm{~d}$, the downstream speed data cannot detect the effect of the incident.

The pattern of the upstream flow time series shown in Figure $2 \mathrm{e}$ is a surprising phenomenon. These time series do not show any difference, regardless of traffic demand, although the measurements appear noisy. The effect of the incident is much more prevalent in the downstream flow time series shown in Figure $2 \mathrm{f}$. Here we can see a decrease in flow rate after the incident occurred. The bigger the traffic demand, the greater the decrease. This phenomenon confirms that due to a decrease in capacity, only part of the traffic demand can be adequately satisfied.

Of course, there is no guarantee that an occurrence of an incident will cause congestion. Incidents only cause problems on a given road segment if the current traffic demand is greater than the capacity of the road segment. For example, on a threelane highway, a pulled over car is often not a problem even with higher traffic demands. Another example could be an accident at night on a three-lane highway that occupies only the outer lane. Although the capacity of the road segment is temporarily reduced, the effect can hardly be detected due to the minimal traffic demand at night. Another extreme is when an incident occurs on an already congested road segment. In this case, it is also not possible to detect an incident simply by taking the detector data into account.

\section{B. Incident detection methods}

An examination of the effects of incidents has shown that the occurrence of an incident distorts the time series of the traffic data in a way that is readily detectable. The challenge is that, depending on the type of incident, the capacity of the road network, the current traffic demand, and the distance between the detectors, the effect appears in the data at a different extent and delay. Because of this, for a truly reliable and rapid detection method it is necessary to carry out our studies on a dataset that is large and contains many different scenarios.

AID methods have been continuously published by researchers since the 1970 s, but the area is still actively researched thanks to the spread of new machine learning methods. In this section, the significant AID methods from professional literature will be described. Since our own method is based on data from traffic detectors, we mainly focused on those methods that use detectors as data sources as well. Of course, methods based on other data sources will also described.

In professional literature, three main metrics are generally used to compare the performance of AID methods. The Detection Rate (DR) represents the ratio of correctly detected 


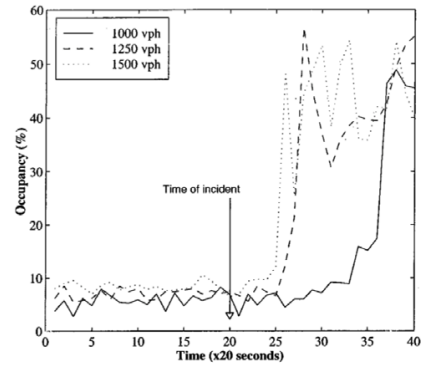

(a) Different occupancy time series measured on the upstream detector.

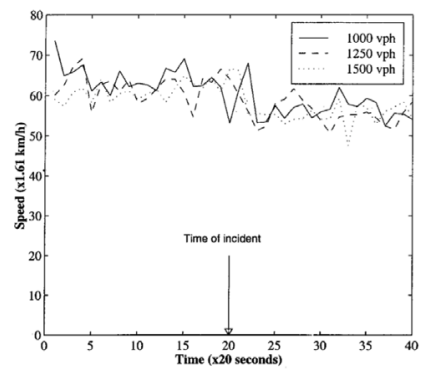

(d) Different speed time series measured on the downstream detector.

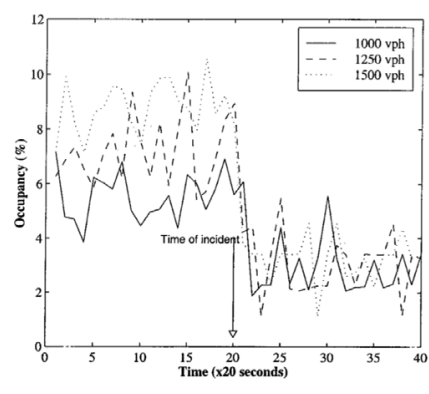

(b) Different occupancy time series measured on the downstream detector.

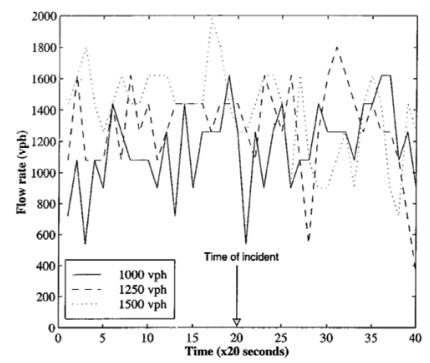

(e) Different dow time series measured on the upstream detector.

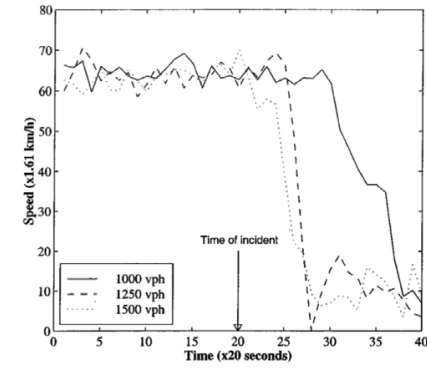

(c) Different speed time series measured on the upstream detector.

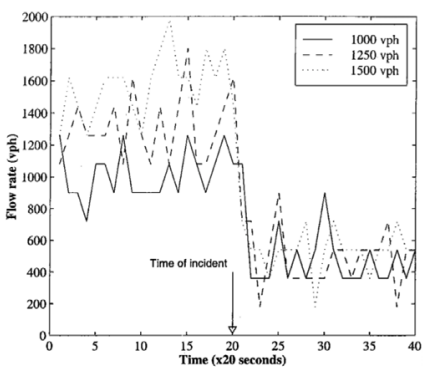

(f) Different dow time series measured on the downstream detector.

Fig. 2: Time series measured on upstream and downstream detectors for different traffic demands [24].

incidents to the total number of incidents. Mean time to detect (MTTD) contains the average amount of time needed to detect incidents. False Alarm Rate (FAR) is the ratio of false incidents detected when no incident actually occurred. The metrics are detailed in Section IV-B.

To make the comparison of methods fair, we have implemented them wherever possible. It is important to point out that the performance of each method was measured on the new incident dataset described in our article, so that the operation of the methods is actually comparable, as each method had to recognize the same incidents. The results are summarized in Table III.

One of the best known of the early AID methods is the California algorithm [26], of which several modified versions have been made [27]. The method compares the occupancy values measured by two adjacent traffic detectors. The steps for comparison are shown in Figure 3, which is from one of the most frequently referenced: the 7th version of the algorithm. The 3 variable denotes the difference between the occupancy values measured on the detectors, the $O C C R D F$ variable denotes the ratio of the difference between occupancy values measured on the detectors, and the $D O C C$ variable denotes the occupancy value of the second detector in the direction of travel.

If these are larger than the pre-set threshold values $T 1$, $T 2, T 3$, then the method considers the measurement to be an incident. Although the method is simple and surprisingly effective, the three thresholds are difficult to adjust. Setting these thresholds incorrectly and using noisy datasets can cause high FAR values. When comparing the results, the algorithm achieved $91.85 \%$ DR, 7.73\% FAR and 7.28-minute MTTD values, which can be considered an average performance.

In order to reduce noise induced FAR, a Low-pass (LP) filter is used in the Minnesota algorithm [28], which is applied separately to the occupancy time series of the two detectors. The time series were examined with disjoint time intervals of 30 seconds. The operation of the algorithm is similar to that of the California algorithm: the steps and two thresholds defined by experts can be used to determine whether an incident had occurred at a given time. The disadvantage of the algorithm is that it cannot distinguish between congestion occurring because of a narrow cross section and actual incidents. Our studies also showed that using the parameter settings proposed in the article, although the DR value was high (99.25\%) and only 2.2 minutes were measured for the MTTD value, the FAR value was extremely high at $48.23 \%$.

Noise-induced difficulties are addressed by the University of California, Berkeley (UCB) algorithm [29] with a cumulative difference in occupancy values.

To do this, first the sum of the occupancy values measured so far on the two adjacent detectors is calculated separately, and then the difference between the two sums is taken. According to the authors, the change in the difference of the cumulative occupancy values follows Random-walk movement, so if the magnitude of movement rises above a pre-set threshold, their method identifies the given time as an incident. Our studies have shown that the method can achieve a low MTTD value of 3.34 minutes and a 3.4\% FAR value, but the value of DR was only $82.22 \%$, which is low compared to other methods. 


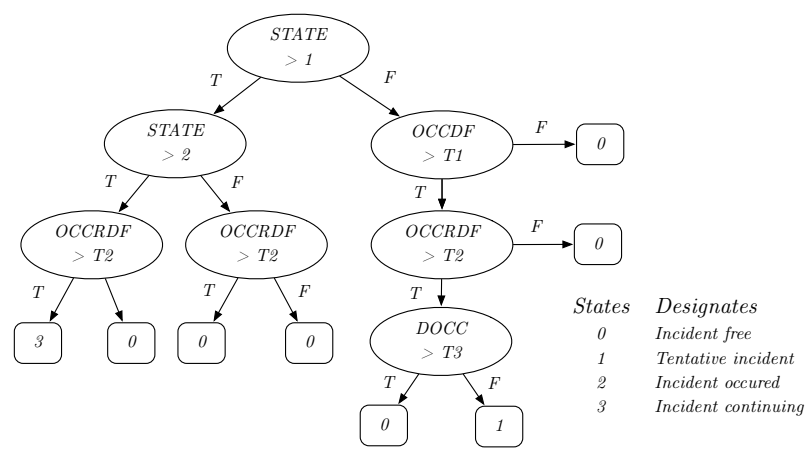

Fig. 3: The tree structure applied in the California algorithm \#7

Other methods use a statistical approach instead of previous solutions. The algorithm of Levin et al [20] uses the value of the $O C C R D F$ variable from the California algorithm for detection. Based on the input dataset, the distribution of the incident and normal measurements are determined separately. In the case of a newly received measurement the Bayesian model is used to determine which distribution the measurement belongs to. Unfortunately, we were unable to reproduce the output of the method because we did not have access to the "Emergency patrol vehicle-assists database" to calculate the probabilities. This database is used to determine the likelihood that an incident will actually affect the capacity of the given road segment. Based on the studies published, the method achieved extremely good results on data with 30second time intervals, but the Mean time to detect (MTTD) value was above 3.5 minutes.

In addition to the previous methods, there are also examples of techniques using different time series prediction for AID implementation in professional literature. In this approach, a prediction model is built for the examined traffic variable, and then monitoring the error of the predictions produced by the model. If this error is above a set threshold value then the given time can be considered an incident. Ahmed et al developed a Auto Regressive Integrated Moving Average (ARIMA) based method [30] that builds models on the occupancy time series of detectors. The $95 \%$ confidence interval of the forecast was used to determine the error limits. Using the confidence interval suggested in the article, we measured a MTTD of 0.98 minutes and a DR of $100 \%$, which are good results, but a FAR value of $8.96 \%$ is considered high. This means almost every 10th alarm is false, which could be troubling for traffic management.

The RoadCast Incident Detection (RCID) [31] algorithm uses the Random Forest (RF) model to build a prediction model for each detector separately. It also incorporates data for holidays and events into the prediction model as an external data source. The integrating external data sources are described in detail in the article. The error limit for the predicted values are determined using the Quantile Random Tree Regression (QRTR) method. The QRTR method is a complement to the $\mathrm{RF}$ model, which estimates the range in which the prediction output will fall based on a known probability value (quantile). If the real measured values are outside the range defined by the quantile three times in a row, the RCID signals an incident. Unfortunately, due to the lack of external data sources, we were unable to reproduce their results, so we could not compare them with the other methods. In their own tests, they achieved very good results, typically with DR values above $80 \%$ and FAR values below $2 \%$, but the MTTD values weren't published.

According to their studies, higher FAR values were measured in cases where an event was held in the area under investigation. In these cases, the forecasts themselves were inaccurate.

The DWT-Logit hybrid method [32] uses binary classification executed with logistic regression to implement AID. The output of the logistic regression indicates the probability of an incident. The probability from which it counts as an incident can be determined by setting a threshold value. The originally noisy data is cleaned with Discrete Wavelet Transformation (DWT), which is used to filter out highfrequency, probably noise-like components. The DWT-Logit hybrid method reached a DR value of $100 \%$ and an MTTD value of 1.19 minutes on our dataset, which is the best result of the examined methods. In contrast, the method's FAR value was extremely high at $27.04 \%$, which is not acceptable in a real system.

In recent years, several publications have tried to achieve break through using Neural Network (NN). The authors of [24] treated the phenomena detected on upstream and downstream detectors separately, building separate Radial Basis Function Neural Network (RBFNN) models for the detectors. Different DWT coefficients were used as inputs for RBFNN models.

For upstream detectors, occupancy and speed coefficients were used, and for downstream detectors occupancy and flow coefficients were taken into account. Simulated and real data were both used for performance analysis. On the real dataset, which contained only 21 incidents, they reached a DR value of $95.2 \%$ and a FAR value of $0 \%$, but the MTTD was not measured, which would have been important information.

Another study [33] focused on correctly setting the hyperparameters of the Neural Network (NN). The method uses fuzzy logic to set the topology of the hidden layers and the parameters of training for the NN. For setting the hidden layers, they used Stacked Auto Encoder (SAE) and Back Progapation (BP). In contrast, Li et al use the less common Extreme Machine Learning (EML) NN for incident detection [34]. The values of speed, flow and occupancy measured on the upstream and downstream detectors are used as inputs for a shared model. According to the author's tests, EML surpassed NN. Although neural network-based methods are promising and the results presented in the publications were better than other machine learning methods, unfortunately the results could not be reproduced in either case, as the publications lacked the precise hyperparameter settings.

In addition to neural networks, another commonly used method is the Support Vector Machine (SVM) [17], [18], [19]. In Motamed's dissertation [17] he developed an incident 
detection method with different SVM parameter settings and using data from two adjacent stations. The SVM model used flow, speed and historical speed values from the upstream detector and flow and speed values from the downstream detector as input parameters. In our comparison, their method reached a $100 \%$ DR value and a 2.76-minute MTTD value, but at the same time we measured a high FAR value of $12.84 \%$.

Nowadays, in addition to traffic detectors, the GPS trajectories measured in vehicles are an increasingly widely used data source. This is often referred to as floating car data. The method developed by $\mathrm{Ki}$ et al [35] uses the phenomenon of high speed differences measured before and after the congestion. For detection they use a Feed Forward Neural Network (FFNN) method with layers $(30,11,2)$. In their next work, they produced a modified version of the previous method [36], which uses layers $(30,14,2)$.

In both cases [35], [36], the data for 3 road segments were examined: before, during, and after the congestion. A binary feature vector with 10 elements was defined for each of the three road segments based on the rate of speed changes measured. To do this, the set of possible values was divided into 10 disjoint ranges, and then the measured ratios were classified into these ranges. The value of an element of the feature vector was 1, where the measured ratio belonged, and 0 in the remaining 9 . The binary feature vectors of the 3 road segments were added sequentially for the input of the neural network. The best of the published results were $77.3 \%$ DR and $8.6 \%$ FAR values. MTTD was not measured.

The Asakura method [23] used Travel Time (TT) instead of speed values for incident detection. The method compares current and previous TT values measured on a road segment in three steps. For each of the three steps a separate preset threshold value is defined, and if it is exceeded the algorithm flags it. Their best results were 50.2\% DR, 0.015\% FAR and 16.1 minutes MTTD values.

The advantage of methods based on GPS trajectories is that there is no need to build infrastructure and thereby serious installation and maintenance costs can be avoided. The disadvantage is that we do not know exactly how many vehicles on the road network we have information on. This value is called penetration. The literature showed that the results were worse than methods using detectors and, additionally, the reliability of the methods may be significantly reduced if the penetration rate is below 5\%. Another problem with the use of GPS trajectories is that companies with large datasets do not make the stored data publicly available in many cases because of commercial or personal legal reasons, which further complicates research. However, trends show that with the development of technology and the spread of new vehicle communication devices [37], [38], this data source will give a new momentum to the research of AID methods.

Looking at the results in Table III, it can be said that the existing methods generally perform well. When examining the DR values, several methods [30], [32], [17] achieved the maximum performance of $100 \%$, and measured relatively low MTTD values. Among the metrics, only the FAR values were too high, and none of the well-performing methods could reach a value below $5 \%$. This is assumed to be due to the fact that models are more sensitive to noise in order to achieve high detection rates and fast detection.

\section{Transient-BASED Automatic Incident DETECTION (TBAID)}

In Section II-B, we saw that the methods currently available in professional literature have achieved good results according to the DR and MTTD metrics. However, it also turned out that the current methods are struggling to manage high FAR values, which can cause major problems for a traffic management center. False alarms can distract controllers from real incidents or cause them to lose focus. In addition, a lot of false alarms can disrupt the operation of the systems that build on the output of the algorithm. The Transient-based Automatic Incident Detection (TBAID) method that we have developed provides a solution to the problem of high FAR values while keeping the DR value high and the MTTD value low.

TBAID uses a new approach to reduce the number of false detections. One of the reasons for the high FAR values found in the current methods is that they want to detect the entire duration of the incidents, which can add extra noise to the training set. In this case, not only the occurrence of the incident, but also the permanent decrease in capacity may also be labeled as an incident, depending on the time at which the traffic authorities specified its end.

During our examination of incident behaviors (see Section II-A), we have noticed that the phenomena of transition between normal and incident states will occur in all cases and is difficult to confuse with other traffic phenomena. Conversely, there may be other reasons for a permanent capacity reduction. Therefore, TBAID focuses on detecting transient phenomena between normal and incident periods instead of the whole incident.

In order to achieve this, in addition to the previously known features describing the collected traffic data, we created new features that have not yet been used in professional literature, focusing specifically on detecting the transient phenomena. For the task of classifying non-incident and incident times, we applied the XGBoost (XGB) model [39], which has not yet been used in the AID area and, according to our studies, has further increased the accuracy of our method.

It is essential for machine learning methods to take into account as many useful features as possible during their operation. A feature is useful when it has sufficient discriminating power, so it can easily separate normal and incident times into two disjoint sets. These new features are produced by transforming raw Speed (SPD), Flow (FLW), and Occupancy (OCC) features measured on the detectors.

\section{A. Occupancy Difference (OCCDF) and Speed Difference (SPDDF)}

The analysis of the incident time series revealed that when an incident occurs, the difference between speed and occupancy values that differs from the average can be observed between upstream and downstream stations. 
Transient-based automatic incident detection method for intelligent transport systems
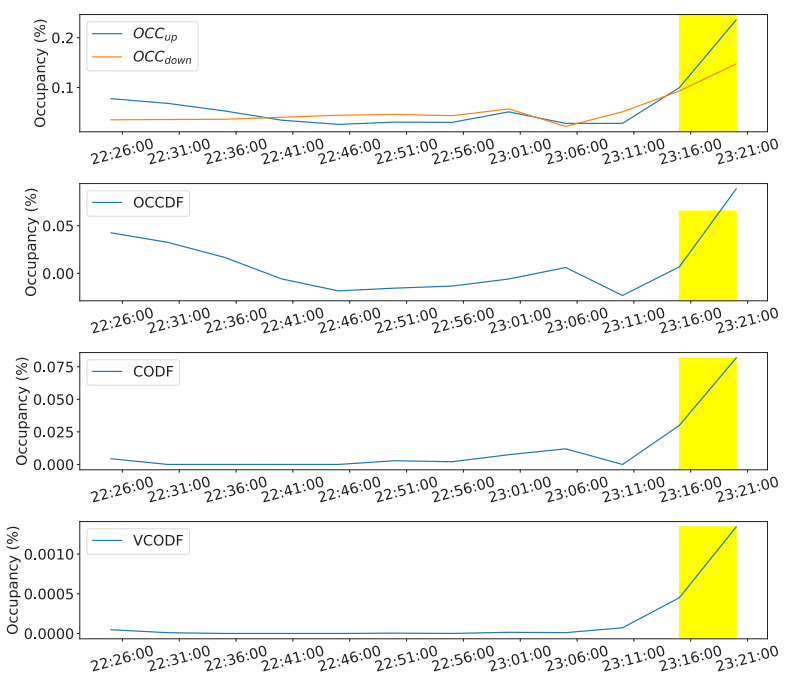

Fig. 4: Features related to occupancy data. The yellow rectangle indicates the presence of the incident.

The OCCDF and SPDDF features represent the differences between upstream and downstream detectors. At times when an incident occurs, the value of the OCCDF and SPDDF features will be higher than the average.

Denote the $N$ long occupancy and speed time series measured on the upstream detector by $\mathrm{OCC}_{u p}=$ $\left\{o c c_{1}, o c c_{2}, \ldots, o c c_{N}\right\}$ and $\mathrm{SPD}_{u p}=\left\{s_{1}, s_{2}, \ldots, s_{N}\right\}$, and denote the $N$ long occupancy and speed time series measured on the downstream detector by $\mathrm{OCC}_{\text {down }}=$ $\left\{\right.$ occ $\left._{1}, o c c_{2}, \ldots, o c c_{N}\right\}$ and $\mathrm{SPD}_{\text {down }}=\left\{s_{1}, s_{2}, \ldots, s_{N}\right\}$.

The time series $\mathrm{OCCDF}=\left\{o c c d f_{1}, o c c d f_{2}, \ldots, o c c d f_{N}\right\}$ is a series of differences between $\mathrm{OCC}_{u p}$ and $\mathrm{OCC}_{\text {down }}$, the $n$th element of which is:

$$
o c c d f_{n}=O C C_{u p, n}-O C C_{d o w n, n}, \quad n=1,2, \ldots, N .
$$

Similar to OCC, the time series $\mathrm{SPDDF}=$ $\left\{s p d d f_{1}, s p d d f_{2}, \ldots, s p d d f_{N}\right\}$ is a series of differences between $\mathrm{SPD}_{u p}$ and $\mathrm{SPD}_{\text {down }}$, the $n$th element of which:

$$
\operatorname{spdd} f_{n}=S P D_{u p, n}-S P D_{\text {down }, n}, \quad n=1,2, \ldots, N .
$$

The second subfigure of Figure 4 shows an example of an OCCDF time series. The time interval affected by the incident was marked with yellow. Although the value of OCCDF increases during the period of the incident in line with the expected behavior, a similar phenomenon is observed at the beginning of the time series, which makes detection difficult and increases the FAR value.

\section{B. Change of Occupancy Difference (CODF) and Change of Speed Difference (CSDF)}

In order to reduce the effects of noise that interfere with detection, as mentioned in the introduction, we focus only on the occurrence of the incidents. During the examination of the incident data, OCCDF and SPDDF values significantly increased as the incident occurred. This observation was used to produce the CODF and CSDF features.

As a first step, the difference between the $n$th and $(n-1)$ th element of the OCCDF time series is determined.

$$
o c h_{i}=\operatorname{occdf}_{n}-\operatorname{occdf}_{n-1},
$$

where $n=2,3, \ldots, N, i=1,2, \ldots N-1$ and $i=$ $n+1$. Then in order to determine time series $\mathrm{CODF}=$ $\left\{\operatorname{cod} f_{1}, \operatorname{cod} f_{2}, \ldots, \operatorname{cod} f_{N-1}\right\}$, all that is left is to examine is whether the value of $o c h_{i}$ is greater than zero:

$$
\operatorname{codf}_{i}= \begin{cases}0 & \text { if } o c h_{i}<0 \\ o c h_{i} & \text { otherwise. }\end{cases}
$$

With this step, we can filter out negative changes that are not related to incidents. The third subfigure of Figure 4 illustrates the impact of this procedure. If we compare this to the second subfigure of Figure 4, which belongs to OCCDF, we can see that the disturbing noise has been significantly reduced.

As with occupancy, we can also define time series CSDF= $\left\{c s d f_{1}, c s d f_{2}, \ldots, c s d f_{N-1}\right\}$ in the case of speed, where:

$$
c s d f_{i}= \begin{cases}0 & \text { if } s c h_{i}<0 \\ s c h_{i} & \text { otherwise }\end{cases}
$$

while:

$$
s c h_{i}=s p d d f_{n}-s p d d f_{n-1},
$$

where $n=2,3, \ldots, N, i=1,2, \ldots N-1$ and $i=n+1$.

\section{Variance of Change of Occupancy Difference (VCODF)}

Although the CODF significantly reduced noise, our goal was to further clean the time series and highlight the phenomenon that is important to us. The time series Variance of Change of Occupancy Difference, $V C O D F=$ $\left\{\operatorname{vod} f_{1}, \operatorname{vodf}_{2}, \ldots, \operatorname{vod}_{N-2}\right\}$ highlights significant changes in the CODF time series by defining sample variance between two consecutive elements. The calculation method for element number $j(j=i+1)$ of the time series VCODF is as follows:

$$
\begin{aligned}
\operatorname{vcodf} f_{j} & =\left(\operatorname{cod} f_{j}-\frac{\operatorname{cod} f_{j}+\operatorname{cod} f_{j-1}}{2}\right)^{2}+ \\
& +\left(\operatorname{cod} f_{j-1}-\frac{\operatorname{cod} f_{j}+\operatorname{cod} f_{j-1}}{2}\right)^{2} .
\end{aligned}
$$

Figure 4 shows that VCODF reduced the noise in the time series compared to the CODF, while the period of the incident still stands out properly.

\section{Difference to Typical Speed (DFTSPD)}

When examining the speed data, another interesting feature is the deviation from historical speed, since the effects of the incident may result in a significant increase in the difference between current and historical data. Let the Typical Speed $(\mathrm{TSPD})=\left\{t s p d_{1}, t s p d_{2}, \ldots, t s p d_{N}\right\}$ be the time series of historical speeds. The $n$th element of an $N$ long DFTSPD time series is:

$$
d f t s p d_{n}=t s p d_{n}-S P D_{u p, n}, \quad n=1,2, \ldots, N,
$$


where $S P D_{u p, n}$ is the $n$th element of the time series measured on the upstream detector, while $t s p d_{n}$ is the $n$th element of the TSPD time series.

During our studies, we found that for the best results, data from the previous two weeks should be considered to determine historical behavior. Where no speed data were available, we used the speed limit for the given road segment.

\section{E. Rolling window (WND) and features squared (SQRD)}

In addition to the new features, we have defined two more optional transformation steps that further increase the reliability of our method.

When examining incidents, an important observation was that after the incident occurred, its effects would not appear immediately in the data. The reason for this is:

- the detectors are usually not installed evenly, so the distances between them vary,

- incidents occur in different positions compared to detectors,

- depending on the type of incident and current traffic, the effect will propagate at different speeds on the road network.

Since the impact of the incident may not appear immediately in the dataset and the magnitude of the effect also depends on the current demand, it is recommended to examine not only a point of time, but time windows in which the impact of the incident is more likely to be detected. To do this, the method we propose uses a fixed sized rolling time window. During training, we tried several time windows of different sizes, of which the 20-minute window size proved to be the best. The rolling window is denoted by $W N D$.

Another optional transformation step is to square the values of the current features. By squaring, normal and incident data become further apart, thereby increasing the descriptive power of the available features, making it easier to detect incidents. Squaring is denoted by $S Q R D$.

\section{Evaluation}

\section{A. Dataset}

During the evaluation, the Caltrans Performance Measurement System (PEMS) dataset [40] was used. The dataset is made up of measurements from approximately 39,000 measuring stations located along major routes in the state of California, USA, going back to 1999 . The size of the dataset is currently about 12 terabytes, which is publicly available and free to download for anyone. Analyzing the total amount of that data would have taken too much time, so we only examined a subset of it. The evaluation was performed in District 3 (Sacramento area) for a one year period from January 1, 2016 to December 31, 2016 and the traffic data was collected by dual loop detectors. Traffic data in the PEMS dataset is available with 30-second and 5-minute aggregation, but the traffic data of 30 -second aggregation was incomplete. Therefore, we were forced to use traffic data with 5-minute aggregation. The advantage of using the 5-minute data is that the data contains significantly less noise than the 30 -second aggregation, but this increases the detection time because the effect of events is displayed with a higher delay.

We also have access to the incidents recorded by California Highway (CHP) via the PEMS Official Site [40]. We used this incident database as a starting point. As many incidents have no effect on traffic data, both automatic and manual filtering steps were required. In the first step, the incidents identified as accidents were selected, because they generally have an impact on traffic trends [12]. In the second step, we selected the incidents where there was a detector nearby and we received data from both detectors. In order to determine the position of the incidents and detectors, the absolute postmile value was used, which represents the distance in miles from the beginning of the road (or from the state border). In step three, we developed a graphical tool to display the 5-minute traffic data at the time of the incident to determine with the naked eye whether the incident caused a change in the measured data.

After the pre-filtering steps, we manually examined more than 5,000 incidents using our graphical tool. When selecting the incidents, we took into account whether, at the time of the incident, there was a visible difference from the historical behavior, a phenomenon described in Section II-A, shown on the detector pair. In many cases, there was no discernible difference between historical and incident data and none of the phenomena described in Section II-A were visible. This may be due to the fact that, depending on its severity, not all incidents have a real impact on the data, and it has also been observed that in case of a detector error, PEMS uses historical data to replace missing periods caused by the error, and therefore such incidents cannot be used. In the end, of the 5,000 incidents, 452 such cases were identified. A detailed description of these steps was published on the official page of our dataset [41].

In the evaluations, we used a randomly selected 60 percent of the incidents as a training dataset and the remaining 40 percent as a test dataset.

\section{B. Metrics}

The evaluation included the most frequently used metrics in professional literature: DR, FAR and MTTD.

Let $\mathcal{I}=\left\{I_{1}, I_{2}, \ldots, I_{|\mathcal{I}|}\right\}$ be the set of incidents in the dataset, and $\hat{\mathcal{I}}=\left\{\hat{I}_{i}\left|\hat{I}_{i} \in \mathcal{I}, i=1,2, \ldots,\right| \hat{\mathcal{I}}|,| \hat{\mathcal{I}}|\leq| \mathcal{I} \mid\right\}$ be the set of detected incidents for which it is true that $\hat{\mathcal{I}} \subseteq$ $\mathcal{I}$. The Detection Rate (DR) represents the ratio of correctly detected incidents to the total number of incidents that can be determined as follows:

$$
D R[\%]=\frac{|\hat{\mathcal{I}}|}{|\mathcal{I}|} \cdot 100,
$$

where $|\mathcal{I}|$ represents the number of incidents and $|\hat{\mathcal{I}}|$ represents the number of detected incidents.

The False Alarm Rate (FAR) shows the ratio of measurements incorrectly detected as incidents to the total number of non-incident measurements:

$$
F A R[\%]=\frac{\# F D T}{\# N I T} \cdot 100,
$$


Enhancing the operational efficiency of

quantum random number generators
TABLE I

THE FEATURES OF THE SCENARIOS USED IN THE EVALUATION.

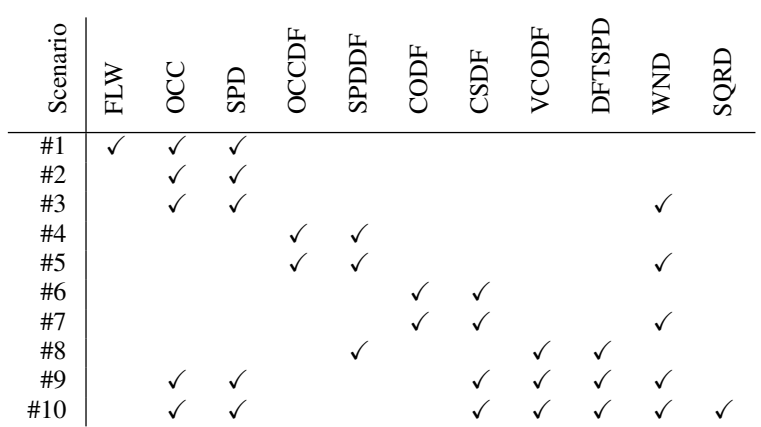

where \#FDT is the number measurements incorrectly detected as incidents and \#NIT is the number of non-incident measurements. A measurement means a time interval, the size of which depends on the dataset.

Mean time to detect (MTTD) determines the average amount of time needed to detect incidents as follows. Let the time of occurrence of the detected incident $\hat{I}_{i}\left(\hat{I}_{i} \in \hat{\mathcal{I}}\right)$ be $\hat{I}_{i, T}$, and the time of detection be $\hat{I}_{i, D T}$. Based on this, the mean detection time of the incidents can be determined as follows:

$$
\operatorname{MTTD}[\text { mins }]=\frac{1}{|\hat{\mathcal{I}}|} \sum_{i=1}^{\hat{\mathcal{I}}} \hat{I}_{i, D T}-\hat{I}_{i, T},
$$

where $|\hat{\mathcal{I}}|$ represents the number of detected incidents.

\section{Scenarios}

Like the method presented by Motamed [17], we have defined several scenarios on the basis of which features have been taken into account. We examined several scenarios in order to compare the results and see if the new features we created actually improved the accuracy of the classification.

The defined scenarios are summarized in Table I. Each column of the table contains the names of the features (or transformations) we use, while each row contains a different scenario. A cell contains an $\checkmark$ icon if the feature is present in the given scenario. Our goal in creating scenarios was to see the FAR decrease caused by the new features we defined.

\section{Results}

After determining the scenarios, in addition to the XGB model, we also trained K-Nearest Neighbor (KNN), SVM and Auto-Encoder Neural Network (AE-NN) classification models using the defined feature, to make sure XGB is indeed one of the best choices for Automatic Incident Detection (AID). It is important to note that the TBAID method ended up using the XGB model and scenario \#10 (Table I).

The hyperparameters of the classification models were set using grid-search optimization, during which a significant number of hyperparameter settings were examined. During the evaluation, nearly 23,000 settings were evaluated to ensure a fair comparison of the results of the models and scenarios.

In evaluating the results, it was a challenge to manage the trade-off between the metrics. To do this, as a first step we set
TABLE II

EVALUATION RESULTS, WHERE 95\% DR, 8\% FAR AND 8-MINUTE MTTD FILTERING CRITERIA WERE APPLIED.

\begin{tabular}{|c|c|c|c|c|c|c|c|c|c|}
\hline \multirow[b]{2}{*}{ 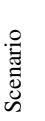 } & \multicolumn{3}{|c|}{ XGB } & \multicolumn{3}{|c|}{ KNN } & \multicolumn{3}{|c|}{ SVM } \\
\hline & $\stackrel{e}{E}$ & 哭 & 齐 & $\stackrel{e}{E}$ & 告 & $\underset{\mathbb{I}}{\stackrel{4}{4}}$ & $\stackrel{\ominus}{\Sigma}$ & 岂 & $\underset{\text { 寽 }}{2}$ \\
\hline$\# 1$ & 4.38 & 94.25 & 3.29 & 6.43 & 95.40 & 3.76 & 3.13 & 96.67 & 6.93 \\
\hline \#2 & 6.28 & 94.25 & 2.71 & 6.78 & 95.98 & 4.76 & nan & nan & nan \\
\hline \#3 & 4.97 & 94.25 & 2.61 & 6.36 & 94.83 & 4.71 & 3.93 & 94.25 & 6.44 \\
\hline \#4 & 5.67 & 94.25 & 6.19 & 7.64 & 94.25 & 5.04 & 3.58 & 97.78 & 7.99 \\
\hline \#5 & 5.03 & 94.25 & 2.67 & 5.86 & 94.25 & 3.58 & nan & nan & nan \\
\hline \#6 & 2.73 & 95.56 & 3.94 & 3.01 & 94.25 & 3.35 & 2.05 & 94.44 & 6.45 \\
\hline \#7 & 4.53 & 94.25 & 1.71 & 6.23 & 94.83 & 3.63 & 3.63 & 94.25 & 5.79 \\
\hline \#8 & 1.98 & 95.56 & 3.74 & 2.09 & 95.56 & 5.44 & nan & nan & nan \\
\hline \#9 & 1.83 & 95.00 & 1.83 & 2.58 & 96.11 & 4.33 & 2.13 & 95.56 & 2.69 \\
\hline$\# 10$ & 2.13 & 95.56 & 0.93 & nan & nan & nan & nan & nan & nan \\
\hline
\end{tabular}

a threshold value for each of the three metrics, below which we did not accept the result.

Then in the second step, the results meeting the criteria were sorted based on the metrics in order of importance of FAR, MTTD, DR.

The results of the evaluation are summarized in Table II. In the case of results presented in Table 2, 95\% DR, $8 \%$ FAR and 8-minute MTTD criteria were applied. These thresholds values can be considered strict, but the model-scenario pairs were generally above predetermined thresholds. With stricter settings, only the XGB model and more complex scenarios performed well. Each column of the table contains the metrics of the models, while the rows are the individual scenarios. In the event that a model-scenario pair did not meet the metric criteria, a "nan" value can be seen in the cell.

Although the evaluations were also carried out for the AENN model, unfortunately in the case of the vast majority we got "nan" values. For this reason, AE-NN is not included in Table II. This does not mean, of course, that AE-NN is a bad model, only that it did not work well with this domain and data.

As seen in Table II, from the point of view of DR none of the models stood out among the models used, as all of them performed similarly. The difference is in MTTD and FAR metrics. Looking at the two metrics, XGB performs visibly better than SVM and KNN models. This is particularly noticeable in scenarios where new features we have developed can be found. For the XGB model, we measured FAR values below $2 \%$ and MTTD values below 2 minutes several times. In scenario \#10, we were able to reach a FAR of even $0.93 \%$, but this resulted in an increase in MTTD value.

It is also worth noting that the values of the MTTD and FAR metrics are constantly improving as we move towards higher-numbered scenarios for every model. This clearly demonstrates that the features we defined can improve the output of MTTD and FAR metrics regardless of the model used.

The following stricter criteria were $97 \%$ DR, 2\% FAR and 2-minute MTTD values. In this case, we no longer created a table, because we did not get evaluable results outside of the XGB - scenario \#10 pair.

This also means that the use of the $S Q R D$ transformation has actually improved the results, since the use of $S Q R D$ is 
TABLE III

METHODS FOUND IN PROFESSIONAL LITERATURE AND TBAID RESULTS ON THE DATASET WE PREPARED. THE TBAID METHOD USES THE XGB MODEL AND SCENARIO \#IO (TABLE I).

\begin{tabular}{clll} 
Algorithm & DR $(\%)$ & FAR $(\%)$ & MTTD (mins) \\
\hline California \#7[26] & 91.85 & 7.73 & 7.28 \\
\hline Minnesota[28] & 99.25 & 48.23 & 2.2 \\
\hline UCB[29] & 82.22 & 3.4 & 3.34 \\
\hline ARIMA-based[30] & 100 & 5.24 & 1.30 \\
\hline DWT-Logit hybrid[32] & 100 & 27.04 & 1.19 \\
\hline Motamed SVM[17] & 100 & 12.84 & 2.76 \\
\hline TBAID & $\mathbf{9 7 . 2 2}$ & $\mathbf{1 . 5 6}$ & $\mathbf{1 . 8 9}$
\end{tabular}

the only difference between scenarios \#9 and \#10. The XGB scenario \#10 pair achieved 97.22\% DR, 1.56\% FAR and 1.89minute MTTD results, which are outstanding when compared to the results of the methods found in professional literature. Although the methods in Table III achieved up to $100 \%$ DR, at the same time very high results were measured for MTTD or FAR values. In contrast, TBAID was able to reach the lowest MTTD-FAR pair at $97 \%$ DR.

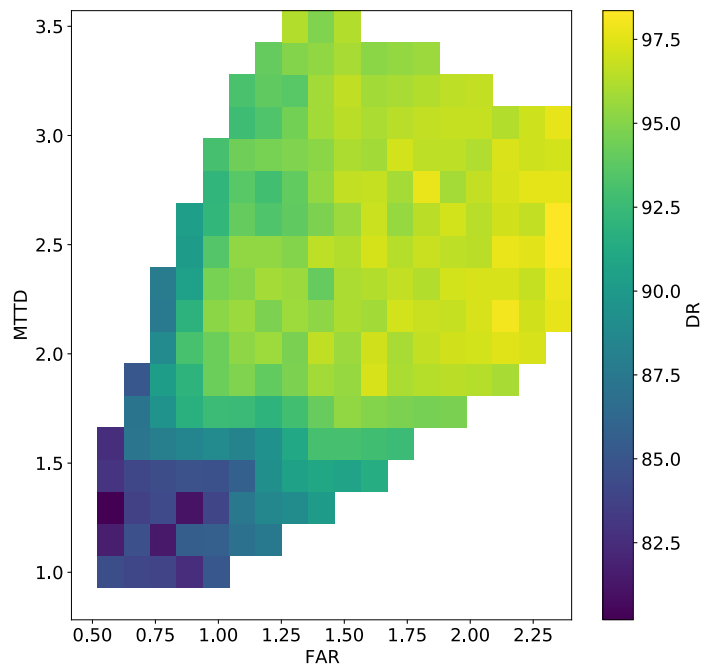

Fig. 5: Change of DR depending on the FAR and MTTD metrics.

We were also curious about the relationships between the change in values for each metric in the case of the XGB model and scenario \#10. To examine this, we used the heatmap shown in Figure 5, in which we plotted DR as a function of FAR and MTTD values with the color scale indicated in the figure. The different results were obtained by changing the hyperparameters of the XGB model and the window size $(W N D)$

Several correlations can be found in Figure 5. When seeking low MTTD and FAR values, the detection rate will also decrease significantly, only reaching values below $90 \%$. If the MTTD is a less important metric, increasing the MTTD value allows for low, less than $1 \%$ FAR values along high DR values above $95 \%$. It is also clear that an increase in FAR typically results in an increase in DR, but this behavior is not true for MTTD. According to Figure 5, the highest DR values can be measured around 2.5 minutes, but after that a slight decrease in DR values can be observed.

Overall, to determine the best result, it is necessary to define a system of criteria (such as the threshold-based solution we use) that determines how important each metric is to us. Generally, if we want to achieve an outstanding result for one of the metrics, it will have a negative effect on the output of the other metrics.

We also considered it important to examine the impact of the window size $(W N D)$ on the metrics. The results of the examination are summarized in the boxplots of Figure 6 . We have examined a total of three window sizes: sizes 2,4 and 6 , which represent 10, 20 and 30 minutes.

The best MTTD and FAR values were clearly measured for window size 2 , but at the same time the DR values were very weak, below $90 \%$, so we do not recommend using window size 2. Conversely, in the case of window size 6 an outstanding DR value can be measured, but the results for MTTD and FAR values are too high. In general, the best results were achieved with window size 4 (20 minutes), which meant relatively low MTTD and FAR values in addition to an about 95\% DR value.

\section{CONCLUSION}

One of the major problems in the transport of major cities is congestion on the road network, which is often caused by unexpected incidents. Quick and accurate detection of incidents can greatly reduce the negative effects they cause.

In this article, we presented the Transient-based Automatic Incident Detection (TBAID) method we developed, which utilizes an approach not yet used in professional literature to detect the occurrence of incidents.

To do this, we first created a new dataset using the PEMS database, which contains 452 incidents and their associated
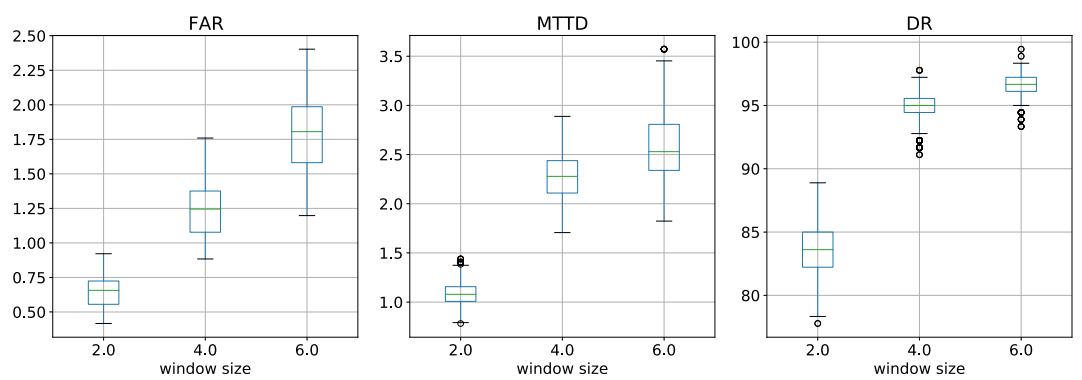

Fig. 6: The impact of window size on each metric. 
traffic data. The dataset has been made publicly available for research purposes.

In addition to the new approach, new features and the XGB model were both used in the TBAID method. The operation of our method has been subject to detailed analysis, in which we have not only compared it with the methods found in professional literature, but also used other classification models in the evaluation of the results. For comparison, we used the standard DR, MTTD and FAR metrics. The TBAID method achieved outstanding 97.22\% DR, 1.56\% FAR and 1.89-minute MTTD values.

In the course of the evaluation, we have also put great emphasis on managing the trade-offs between the metrics and understanding how the performance of our method changes by setting different metric criteria.

\section{REFERENCES}

[1] J. B. Bump, S. K. Reddiar, and A. Soucat, "When do governments support common goods for health? four cases on surveillance, traffic congestion, road safety, and air pollution," Health Systems \& Reform, vol. 5, no. 4, pp. 293-306, 2019, Dor: 10.1080/23288604.2019.1661212.

[2] N. Zhong, J. Cao, and Y. Wang, "Traffic congestion, ambient air pollution, and health: Evidence from driving restrictions in beijing," Journal of the Association of Environmental and Resource Economists, vol. 4, no. 3, pp. 821-856, 2017, DoI: 10.1086/692115.

[3] O. K. Kurt, J. Zhang, and K. E. Pinkerton, "Pulmonary health effects of air pollution," Current opinion in pulmonary medicine, vol. 22, no. 2, p. 138, 2016, DoI: 10.1097/MCP.0000000000000248.

[4] K. Chen, A. Schneider, J. Cyrys, K. Wolf, C. Meisinger, M. Heier, W. von Scheidt, B. Kuch, M. Pitz, A. Peters et al., "Hourly exposure to ultrafine particle metrics and the onset of myocardial infarction in augsburg, germany," Environmental Health Perspectives, vol. 128, no. 1, p. 017003, 2020, DoI: 10.1289/EHP5478.

[5] X. Tian, H. Dai, Y. Geng, J. Wilson, R. Wu, Y. Xie, and H. Hao, "Economic impacts from pm2. 5 pollution-related health effects in china's road transport sector: A provincial-level analysis," Environment international, vol. 115, pp. 220-229, 2018, Dor: 10.1016/j.envint.2018.03.030.

[6] L. Zhu, F. R. Yu, Y. Wang, B. Ning, and T. Tang, "Big data analytics in intelligent transportation systems: A survey," IEEE Transactions on Intelligent Transportation Systems, vol. 20, no. 1, pp. 383-398, 2018, DoI: $10.1109 /$ TITS.2018.2815678

[7] J. Li, Y. Zhang, and Y. Chen, "A self-adaptive traffic light control system based on speed of vehicles," in 2016 IEEE International Conference on Software Quality, Reliability and Security Companion (QRS-C). IEEE, 2016, pp. 382-388, Dor: 10.1109/QRS-C.2016.58.

[8] R. W. Hall, "Non-recurrent congestion: How big is the problem? are traveler information systems the solution?" Transportation Research Part C: Emerging Technologies, vol. 1, no. 1, pp. 89-103, 1993, DoI: 10.1016/0968-090X(93)90022-8.

[9] A. Skabardonis, P. Varaiya, and K. F. Petty, "Measuring recurrent and nonrecurrent traffic congestion," Transportation Research Record, vol. 1856, no. 1, pp. 118-124, 2003, Dor: 10.3141/1856-12.

[10] J. Evans, B. Waterson, and A. Hamilton, "Evolution and future of urban road incident detection algorithms," Journal of Transportation Engineering, Part A: Systems, vol. 146, no. 6, p. 03120001, 2020, DoI: $10.1061 /$ JTEPBS.0000362.

[11] H. Zhang and A. Khattak, "What is the role of multiple secondary incidents in traffic operations?" Journal of Transportation Engineering, vol. 136, no. 11, pp. 986-997, 2010, Dor: 10.1061/(ASCE)TE.1943-5436.0000164.

[12] N. Owens, A. Armstrong, P. Sullivan, C. Mitchell, D. Newton, R. Brewster, and T. Trego, "Traffic incident management handbook," Tech. Rep., 2010.

[13] T. Agenda, "Manual on uniform traffic control devices," 2017.

[14] R. J. Javid and R. J. Javid, "A framework for travel time variability analysis using urban traffic incident data," IATSS research, vol. 42, no. 1, pp. 30-38, 2018, Dor: 10.1016/j.iatssr.2017.06.003.
[15] A. T. Hojati, L. Ferreira, P. Charles, M. R. bin Kabit et al., "Analysing freeway traffic-incident duration using an australian data set," Road \& Transport Research: A Journal of Australian and New Zealand Research and Practice, vol. 21, no. 2, p. 19, 2012.

[16] Z. Chen and W. Fan, "Data analytics approach for travel time reliability pattern analysis and prediction," Journal of Modern Transportation, vol. 27, no. 4, pp. 250-265, 2019, Dor: 10.1007/s40534-019-00195-6.

[17] M. Motamed et al., "Developing a real-time freeway incident detection model using machine learning techniques," Ph.D. dissertation, 2016.

[18] Y. Sun and Z. Hou, "A novel abnormal traffic incident detection method based on improved support vector machine," Journal of Applied Science and Engineering, vol. 21, no. 1, pp. 45-50, 2018, Dor: 10.6180/jase.201803_21(1).0006.

[19] J. Xiao, "Svm and knn ensemble learning for traffic incident detection," Physica A: Statistical Mechanics and its Applications, vol. 517, pp. 29-35, 2019, Dor: 10.1016/j.physa.2018.10.060.

[20] M. Levin and G. M. Krause, "Incident detection: A bayesian approach," Transportation Research Record, vol. 682, pp. 52-58, 1978.

[21] Y. Hernandez-Potiomkin, M. Saifuzzaman, E. Bert, R. Mena-Yedra, T. Djukic, and J. Casas, "Unsupervised incident detection model in urban and freeway networks," in 2018 21st International Conference on Intelligent Transportation Systems (ITSC). IEEE, 2018, pp. $1763-$ 1769, DoI: 10.1109/ITSC.2018.8569642.

[22] E. D'Andrea and F. Marcelloni, "Detection of traffic congestion and incidents from gps trace analysis," Expert Systems with Applications, vol. 73, pp. 43-56, 2017, Dor: 10.1016/j.eswa.2016.12.018.

[23] Y. Asakura, T. Kusakabe, L. X. Nguyen, and T. Ushiki, "Incident detection methods using probe vehicles with on-board gps equipment," Transportation research part C: emerging technologies, vol. 81, pp. 330-341, 2017, DoI: 10.1016/j.trc.2016.11.023.

[24] A. Karim and H. Adeli, "Incident detection algorithm using wavelet energy representation of traffic patterns," Journal of Transportation Engineering, vol. 128, no. 3, pp. 232-242, 2002, DoI: $10.1061 /(A S C E) 0733-947 X(2002) 128: 3(232)$.

[25] N. H. Gartner, C. J. Messer, and A. Rathi, "Traffic flow theory-a stateof-the-art report: revised monograph on traffic flow theory," 2002.

[26] H. Payne, E. Helfenbein, and H. Knobel, "Development and testing of incident detection algorithms, volume 2: Research methodology and detailed results," Tech. Rep., 1976.

[27] M. Levin and G. M. Krause, "Incident-detection algorithms part 1. offline evaluation," Transportation Research Record, vol. 722, pp. 49$58,1979$.

[28] Y. J. Stephanedes and A. P. Chassiakos, "Application of filtering techniques for incident detection," Journal of transportation engineering, vol. 119, no. 1, pp. 13-26, 1993, DoI: 10.1061/(ASCE)0733-947X(1993)119:1(13).

[29] W.-H. Lin and C. F. Daganzo, "A simple detection scheme for delayinducing freeway incidents," Transportation Research Part A: Policy and Practice, vol. 31, no. 2, pp. 141-155, 1997, Dor: 10.1016/S0965-8564(96)00009-2.

[30] S. Ahmed and A. R. Cook, "Application of time-series analysis techniques to freeway incident detection," Transportation Research Record, vol. 841, pp. 19-21, 1982.

[31] J. Evans, B. Waterson, and A. Hamilton, "A random forest incident detection algorithm that incorporates contexts," International Journal of Intelligent Transportation Systems Research, pp. 1-13, 2019, DOI: 10.1007/s13177-019-00194-1.

[32] S. Agarwal, P. Kachroo, and E. Regentova, "A hybrid model using logistic regression and wavelet transformation to detect traffic incidents," Iatss Research, vol. 40, no. 1, pp. 56-63, 2016, DoI: 10.1016/j.iatssr.2016.06.001.

[33] C. El Hatri and J. Boumhidi, "Fuzzy deep learning based urban traffic incident detection," Cognitive Systems Research, vol. 50, pp. 206-213, 2018, Dor: $10.1016 /$ j.cogsys.2017.12.002.

[34] L. Li, X. Qu, J. Zhang, and B. Ran, "Traffic incident detection based on extreme machine learning," Journal of Applied Science and Engineering, vol. 20, no. 4, pp. 409-416, 2017, DoI: $10.6180 /$ jase.2017.20.4.01 
[35] Y.-K. Ki, N.-W. Heo, J.-W. Choi, G.-H. Ahn, and K.-S. Park, "An incident detection algorithm using artificial neural networks and traffic information," in 2018 Cybernetics \& Informatics (K\&I). IEEE, 2018, pp. 1-5, DOI: 10.1109/CYBERI.2018.8337551.

[36] Y.-K. Ki, W.-T. Jeong, H.-J. Kwon, and M.-R. Kim, "An algorithm for incident detection using artificial neural networks," in 2019 25th Conference of Open Innovations Association (FRUCT). IEEE, 2019, pp. 162-167, DoI: 10.23919/FRUCT48121.2019.8981509.

[37] N. Varga, L. Bokor, A. Takács, J. Kovács, and L. Virág, "Anarchitecture proposal for $\mathrm{v} 2 \mathrm{x}$ communication-centric traffic light controller systems," in 2017 15th International Conference on ITS Telecommunications (ITST). IEEE, 2017, pp. 1-7, Dor: 10.1109/ITST.2017.7972217.

[38] Á. Knapp, A. Wippelhauser, D. Magyar, and G. Gódor, "An overview of current and future vehicular communication technologies," Periodica Polytechnica Transportation Engineering, 2020, DoI: 10.3311/PPtr.15922.

[39] T. Chen and C. Guestrin, "Xgboost: A scalable tree boosting system," in Proceedings of the 22nd acm sigkdd international conference on knowledge discovery and data mining, 2016, pp. 785-794, DoI: $10.1145 / 2939672.2939785$.

[40] "Caltrans pems," 2020, [Online; accessed 17-March-2020]. [Online]. Available: http://pems.dot.ca.gov/

[41] "Traffic incident dataset," 2020, [Online; accessed 14-September-2020]. [Online]. Available: https://gitlab.medianets.hu/anagy/incident_dataset

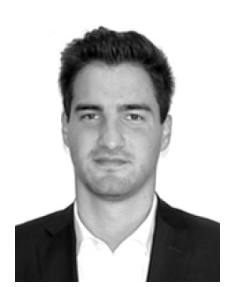

Attila M. Nagy received the B.S. and M.S. degrees in computer engineering from the Budapest University of Technology and Economics (BME), Budapest, in 2016. From 2016-2020, he was PhD student in computer engineering from the Budapest University of Technology and Economics (BME), Budapest. Since 2020 he is a Research Assistant in MEDIANETS laboratory at Department of Networked Systems and Services, BME. His research interests include time series data mining and analysis, traffic prediction, traffic congestion data analysis, automatic traffic incident detection.

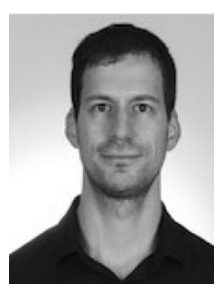

Bernát Wiandt received his $\mathrm{PhD}$ from the Budapest University of Technology and Economics (BME) in 2017. Currently he is an Assistant Professor at the Department of Networked Systems and Services, Member of the Multimedia Networks and Services Laboratory.

$\mathrm{He}$ has done research on self-organizing systems, flocking and distributed task allocation, recently his research interests include machine learning and data analytics for smart cities and intelligent transportation management systems.

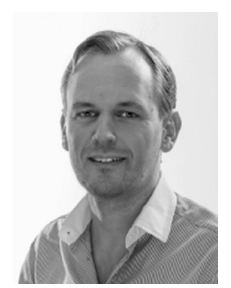

Vilmos Simon received his $\mathrm{PhD}$ from the Budapest University of Technology and Economics (BME) in 2009. Currently he is an Associate Professor at the Department of Networked Systems and Services, Head of the Multimedia Networks and Services Laboratory and Deputy Head of Department of Networked Systems and Services.

He has done research on mobility management and energy efficiency in mobile cellular systems and selforganized mobile networks, recently his research interests include machine learning and data analytics for smart cities and intelligent transportation management systems. He published $50+$ papers in international journals and conferences, and acts as a reviewer or organizer for numerous scientific conferences. He serves currently as the Corporate liaison vice president for the Connected and Automated Mobility Cluster of Zala. 